\title{
Transcolonic Endoscopic Ultrasound-Guided Fine-Needle Aspiration Has a Promising Future
}

\author{
Sharmila Sachithanandan \\ Department of Gastroenterology, Ramsay-Sime Darby Medical Centre, Subang Jaya, Kuala Lumpur, Malaysia
}

See "Utility of Forward-View Echoendoscopy for Transcolonic Fine-Needle Aspiration of Extracolonic Lesions: An Institutional Experience" by Nithi Thinrungroj, Kazuo Hara, Nobumasa Mizuno, et al., on page 60-64.

The use of endoscopic ultrasound (EUS)-guided fine-needle aspiration or EUS-guided fine-needle biopsy to obtain tissue for histological examination from extraluminal lesions in close proximity to, or within, the walls of the gastrointestinal tract has long been well established. This has considerably influenced the staging of esophageal, gastric, rectal, pancreatic, and hepatobiliary cancers. In addition, EUS has become an indispensable diagnostic tool in distinguishing benign from malignant lesions in the aforementioned anatomical areas. Progress in this field has accelerated with interventional EUS, as exemplified by procedures such as celiac plexus neurolysis, pancreatic pseudocyst drainage, access to the biliary tree in failed endoscopic retrograde cholangiopancreatography, and implantation of fiducial markers and radioactive seeds in malignant tumors. ${ }^{1}$ In stark contrast, the application of this technique to fulfill even the most basic need of obtaining biopsy specimens from lesions in the lower abdomen and pelvis by intubation of the colon has been sporadic at best. This has been attributed to the intrinsic difficulties in negotiating the oblique-viewing curvilinear array EUS (CLA-EUS) echoendo-

Received: December 27, 2019 Revised: January 13, 2020

Accepted: January 16, 2020

Correspondence: Sharmila Sachithanandan

Department of Gastroenterology, Ramsay-Sime Darby Medical Centre, Subang Jaya, Kuala Lumpur, Malaysia

Tel: +60-1-2606-8979, Fax: +60-3-5639-1313, E-mail: sharmilasachi68@gmail. com

ORCID: https://orcid.org/0000-0001-7033-3992

(cc) This is an Open Access article distributed under the terms of the Creative Commons Attribution Non-Commercial License (http://creativecommons.org/ licenses/by-nc/3.0) which permits unrestricted non-commercial use, distribution, and reproduction in any medium, provided the original work is properly cited. scope through the colon.

In this issue of Clinical Endoscopy, Thinrungroj et al. ${ }^{2}$ reported their institutional experience of obtaining transcolonic endoscopic ultrasound-guided fine-needle aspiration specimens of extracolonic lesions in the pelvis and lower abdomen, providing grounds for optimism that the technical difficulties that have discouraged many in the past are not insurmountable.

In this retrospective review of 13 consecutive patients covering a period of only $>3$ years, the diagnostic performance of transcolonic endoscopic ultrasound-guided fine-needle aspiration in detecting malignant lesions was shown to be highly impressive, with a sensitivity, specificity, and accuracy of $91 \%, 100 \%$, and $92 \%$, respectively. The authors attributed the favorable results to two key elements. The first was the use of a forward-viewing EUS (FV-EUS) scope that enabled easier intubation and navigation of the colon than with CLA-EUS, together with the lower attendant risk of perforation. The second was the use of fluoroscopy with a C-arm X-ray position to determine whether the position of the tip of the scope was close to the site of the target lesion, as seen on imaging modalities such as computed tomography, positron emission tomography-computed tomography, or magnetic resonance imaging. The use of fluoroscopy mitigated, to some extent, the narrower ultrasonic scanning range $\left(90^{\circ}\right)$ afforded by FV-EUS.

Historically, only two other studies have used FV-EUS for pericolonic lesions, neither of which employed fluoroscopy. The first study by Nguyen-Tang et al. sampled subepithelial lesions within the colon. ${ }^{3}$ The second study by Uchida et al. described the feasibility of transcolonic endoscopic ultra- 
sound-guided fine-needle aspiration in only two patients. ${ }^{4}$ As the scanning range of FV-EUS is only half that of CLA-EUS $\left(180^{\circ}\right)$, fluoroscopy was used to help obtain the optimal position for puncture.

As Thinrungroj et al. pointed out, this study had the limitation of a small sample size. ${ }^{2}$ In addition, the preponderance of left-sided pericolonic lesions precludes extrapolating the success rates to lesions on the right. It is rather likely that this study was conducted in a center with a high level of technical expertise, which raises the question of whether the results are reproducible in most other centers. Prospective multicenter studies with a large number of cases are needed before this technique can become standard of care. Notwithstanding, Thinrungroj et al. have made the strongest case thus far that the design and execution of such studies are indeed justified. ${ }^{2}$
Conflicts of Interest

The author has no financial conflicts of interest.

\section{REFERENCES}

1. Valero M, Robles-Medranda C. Endoscopic ultrasound in oncology: an update of clinical applications in the gastrointestinal tract. World J Gastrointest Endosc 2017;9:243-254.

2. Thinrungroj N, Hara K, Mizuno N, Kuwahara T, Okuno N. Utility of forward-view echoendoscopy for transcolonic fine-needle aspiration of extracolonic lesions: an institutional experience. Clin Endosc 2020;53:60-64.

3. Nguyen-Tang T, Shah JN, Sanchez-Yague A, Binmoeller KF. Use of the front-view forward-array echoendoscope to evaluate right colonic subepithelial lesions. Gastrointest Endosc 2010;72:606-610.

4. Uchida N, Galasso D, Seerden TC, et al. EUS-FNA of extracolonic lesions by using the forward-viewing linear echoendoscope. Gastrointest Endosc 2010;72:1321-1323. 